A. K. Dupree and A. O. Benz, Eds.

\title{
Waves in the Solar Corona
}

\author{
Eckart Marsch \\ Max-Planck-Institut für Aeronomie, 37191 Katlenburg-Lindau, Germany
}

\begin{abstract}
Waves at all scales, ranging in wavelength from the size of a loop (fraction of a solar radius) down to the gyroradii of coronal ions (about hundred meters), are believed to play a key role in the transport of mechanical energy from the chromosphere to the Sun's corona and wind, and through the dissipation of wave energy in the heating and sustaining of the solar corona. A concise review of new observations and theories of waves in the magnetically confined (loops) as well as open (holes) corona is given. Evidence obtained from spectroscopy of lines emitted by coronal ions points to cyclotron resonance absorption as a possible cause of the observed emission-line broadenings. Novel remote-sensing solar observations reveal low-frequency loop oscillations as expected from MHD theory, which appear to be excited by magnetic activity in connection with flares and to be strongly damped. Kinetic models of the corona indicate the importance of wave-particle interactions that hold the key to understand ion acceleration and heating by high-frequency waves.
\end{abstract}

\section{Introduction}

This concise review addresses selected current issues of waves in the solar corona. We start with describing basic coronal magnetic field structures, in which the waves and flows occur. Coronal loops and funnels are the building blocks of the lower corona, and their fields guide the distribution and propagation of wavemechanical energy which is generated by steady photospheric magnetoconvection and transient activity of the magnetic network. Kinetic plasma waves and ion heating by them are addressed, as well as the magnetohydrodynamic oscillations of loops. Finally some ideas about the generation, steepening and dissipation of coronal waves and turbulence are presented.

\section{Coronal magnetic field structures}

Waves in the solar corona is a difficult subject, because the corona is known to be highly structured and nonuniform (see the early work of Roberts, Edwin \& Benz 1984, and Roberts 1985 on magnetohydrodynamic (MHD) waves). Furthermore, the direct measurement of the magnetic field vector in the corona is still not possible. We have for the corona to rely on models of the magnetic field, obtained by extrapolation (through potential-field, force-free, or MHD numerical methods) from the bottom of the corona, whereas at the photosphere/chromosphere 
interface the magnetic field vector can routinely be measured by means of full Stokes polarimetry or its single components by the simple Zeeman effect. Only very recently, the magnetic vector at the coronal base was measured directly (Solanki et al. 2003), which led to the detection of a current sheet and illustrated the topological change of the field with height in the transition region. Modern extreme ultraviolet (EUV) imagers on SOHO and TRACE provide "images" of the field, which is made visible through the emission of plasma confined therein.

Such images reveal bright closed loops (on all scales down to $1^{\prime \prime}$ ) and streamers, and in striking contrast dark voids, the coronal funnels and coronal holes (CHs). The chromospheric and lower-transition-region emissions (at temperatures below about $10^{5} \mathrm{~K}$ ) exhibit the magnetic network with typical cell sizes of 20-30 Mm. Together these magnetic structures constitute a complex background field, in which the waves (either kinetic or fluid-type and linear or nonlinear) are excited (e.g., by reconnection or foot-point motion in the photosphere), and through which they have to propagate, while escaping on open or bouncing on closed field lines.

\section{Waves and flows in loops and funnels}

It is difficult to observe coronal waves, because of the incomplete and insufficient plasma diagnostics possible in the solar atmosphere. In-situ measurements, such as made in other solar-system plasmas (e.g. in the solar wind or Earth's magnetosphere), are impossible in the corona. Only remote-sensing is feasible, directly by using photons (in X-rays, ultraviolet, visible, and infrared light), or electromagnetic waves (radio, plasma waves), and somewhat more indirectly by means of corpuscular radiation (solar wind and energetic particles). Waves manifest themselves in spectral lines through intensity (i.e., electron density) modulation and the Doppler effect, which leads to line shifts being either resolved as flows or unresolved as thermal and turbulent broadenings. However, spectroscopy suffers from the line-of-sight (LOS) problem, which requires even for optically thin lines to disentangle the integrated signal along the LOS, and from the radiative source and transfer problem, which implies to consider conditions far from collisional equilibrium for the corona.

Concerning the largest resolvable wave scales, it is required that the wavelength $\lambda<L$ and period $P<T$, where $L$ is the extent of the field of view and $T$ the duration of an observational sequence. This requirement is readily fulfilled by modern instruments. Also, their spectral resolutions are sufficient to measure Doppler shifts and broadenings. The integrated effects of the resolved low-frequency waves or the unresolved high-frequency turbulence are subsumed observationally in the line-of-side turbulent amplitude $\xi$, which leads to an effective temperature of an ion (with kinetic temperature $T_{i}$ and mass $m_{i}$ ) according to the formula ( $k_{B}$ is the Boltzmann constant):

$$
T_{i, e f f}=T_{i}+\frac{m_{i}}{2 k_{B}} \xi^{2}
$$

Ample evidence was in the recent past provided by the TRACE and SOHO missions for the existence of waves trapped in loops or propagating in flux tubes 


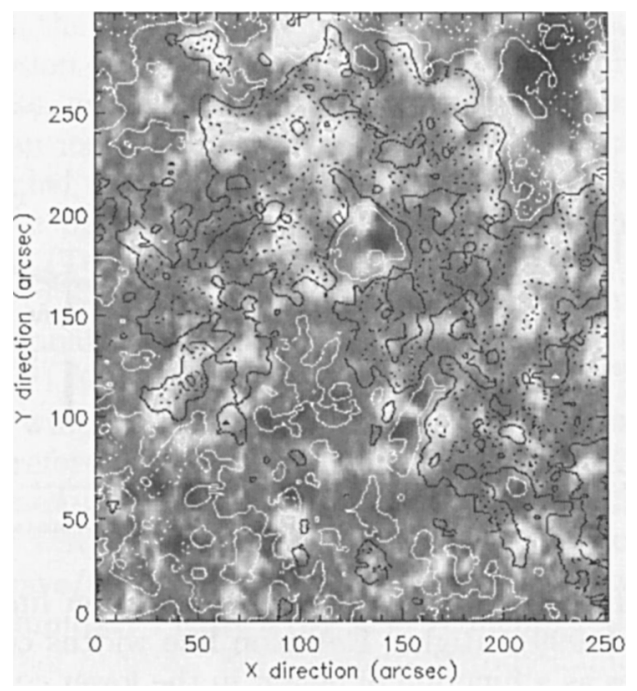

Figure 1. Photospheric magnetogram and neon-ion outflow inferred from SUMER observations of Doppler blue-shifts of Ne VIII $(\lambda 77 \mathrm{~nm})$, relative to $\mathrm{C}$ I $(\lambda 154.2 \mathrm{~nm})$ in an equatorial coronal hole. Black contours: dotted $10 \mathrm{~km} / \mathrm{s}$, solid, $7 \mathrm{~km} / \mathrm{s}$; white: solid $3 \mathrm{~km} / \mathrm{s}$. Kitt Peak magnetogram: white, positive, black, negative field polarity (Xia, Marsch, \& Curdt 2003). Note the close correlation between blueshift and positive field, indicating significant upflows in open unipolar magnetic regions.

(see, e.g., Aschwanden et al. 2002; Wang 2003 for a review) and plasma flowing out in open funnels; see Xia et al. (2003) and the results shown in Figure 1. Coronal funnels and their associated ion flows have been modelled by Marsch \& Tu (1997) and Hackenberg, Marsch \& Mann (1999). In their two-fluid model they considered ion heating that is assumed to be caused by cyclotron-wave sweeping. A steep inward temperature gradient resulting from electron heat conduction was also found. The sonic point is located close to the sun at $2 R_{s}$ $\left(1 R_{s}=700 \mathrm{Mm}\right)$.

Theoretically, coronal waves confined in loops are expected to travel from loop top to bottom, when being excited at the loop apex, and thus will produce mainly redshifts (Hansteen et al. 1993). On the contrary, the plasma streaming out on open field lines is supposed to produce mainly blueshifts. Concerning the observational evidence, both expected types of Doppler shift have indeed been observed (Hassler et al. 1999; Wilhelm et al. 2000; Xia et al. 2003; Seely et al. 1997; Tu et al. 1998; Peter \& Judge 1999; Peter 2001; and further references therein). A survey of the observational situation is given in the subsequent Figure 2, which shows in its left and right hand panel the line shifts and widths (separately for the core and wing parts), respectively versus the line-formation temperature, i.e. essentially the electron temperature in the range from $10^{4}$ to $10^{6} \mathrm{~K}$, values corresponding to lower (below $1.3 R_{s}$ ) heights in the solar corona. 

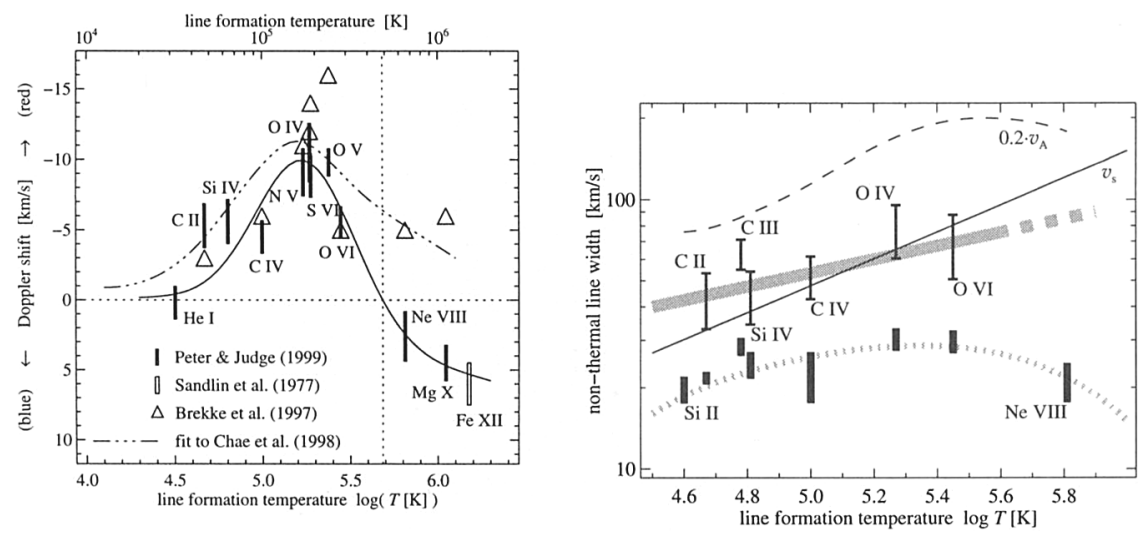

Figure 2. Left: Doppler shifts of EUV emission lines according to Peter \& Judge (1999). Right: Emission line widths corresponding to wave amplitudes as a function of height in the lower corona after Peter (2001). The widths of the core and wing parts of each line are plotted separately. Alfvén speed and sound speed are given as well.

Note the distinct change from red to blue shifts in Figure 2 (left frame) with increasing temperature.

Considering the right frame of Figure 3, note further the clear separation in line width between the core and tail components of all the lines shown, with the width ranging between 20 and $30 \mathrm{~km} / \mathrm{s}$ in the core (lower points) but steadily growing with height in the corona from about 40 to $90 \mathrm{~km} / \mathrm{s}$ in the line tail (upper points). The major contributions, to perhaps the core but certainly the tail component, come from $\xi$ which is an empirical amplitude conveniently used to estimate the amount of turbulent wave energy existing in the corona (see formula (1) again).

\section{Coronal plasma waves and ion heating}

Let us now concentrate on plasma waves. Whereas low-frequency fluid modes in the corona can easily be resolved, it is presently not possible to detect highfrequency MHD and plasma waves (with frequencies above $1 \mathrm{~Hz}$ ). To do this the spatial (pixel size) and temporal (exposure/cadence) resolution of the observations must be less than the wave lengths and periods, a requirement which is missed by the instrumentation on SOHO and TRACE by orders of magnitude.

The shortest relevant ion kinetic scales are: Proton inertial length or the wavelength of a cyclotron wave, which is $\lambda_{p}=2 \pi v_{A} / \Omega_{p}=1434\left(\mathrm{n} / \mathrm{cm}^{3}\right)^{-1 / 2} \mathrm{~km}$ (about $100 \mathrm{~m}$ in a CH), its period $P_{p}=2 \pi / \Omega_{p}=0.66(B / \mathrm{G})^{-1} \mathrm{~ms}$, and the resonant wave amplitude, $\delta V_{g}$, which may be less than $1 \mathrm{~km} / \mathrm{s}$, i.e. $\delta V_{g} \leq$ $0.001 v_{A}$. Here $\Omega_{i}=e_{i} B /\left(m_{i} c\right)$ is the gyro frequency of species $i$ with charge $e_{i}$, $B$ the magnetic field, and $v_{A}$ the Alfvén speed.

Heavy ion temperatures have been estimated from measured line widths by means of equation (1). The ion cyclotron resonance frequency is $f_{i} / \mathrm{kHz}=$ 
$1.5 Z_{i} /\left(A_{i} B / \mathrm{G}\right)$, with the ionic charge number, $Z_{i}$, and atomic mass number, $A_{i}$. By resonance absorption of waves near the gyro frequency, the magnetic moment of an ion will increase, and consequently the perpendicular ion temperature as well, especially for an ion moving in the declining field (mirror configuration) of an expanding funnel or $\mathrm{CH}$. This effect may lead to a scaling of the line widths with the wave period, i.e. $P_{i} \sim A_{i} / Z_{i}$, which was indeed found from SUMER observations (Tu et al. 1998; Tu et al. 1999). The resulting ion kinetic temperature is $T_{i}=(2-6) \mathrm{MK}$ at $r=1.15 R_{s}$. By comparison with the small length $\lambda_{p}$, the collisional free path, $\lambda_{c} \approx 3\left(T_{6}^{2} / n_{10}\right) \mathrm{km}$ (with $T_{6}=T / 10^{6} \mathrm{~K}$ and $n_{10}=n / 10^{10} \mathrm{~cm}^{3}$ ), for a proton is rather large in the corona. In a $\mathrm{CH}$ we find $\lambda_{c} \approx 1000 \mathrm{~km}$, which is about the pixel size of present EUV imagers and spectrometers. Therefore, the corona should largely behave like a collisionless and tightly magnetized plasma (because of the low plasma beta of only a few $\%$ ), and thus kinetic wave effects will dominate over collisional effects.

The empirical wave/turbulence amplitude $\xi$ was shown versus line-formation temperature in the summary Figure 2 (see also Wilhelm et al. (1998) and Marsch et al. (1997) for further results). For magnetohydrodynamic waves travelling on open field lines we expect, that when they escape without damping or suffer only weak dissipation, their amplitude follows a WKB-type evolution. This means a scaling like $\xi \sim \rho^{-1 / 4}$ for Alfvén waves, which seems consistent with the results presented in the upper curve in the right panel of Figure 2. We expect that waves confined in loops are more prone to strong dissipation with consequent loop heating than the ones escaping in funnels. This might explain why there is a maximum in the lower curve in the right panel.

High-frequency (above $1 \mathrm{~Hz}$ at the base) waves propagating to the outer corona may finally dissipate within a few $R_{s}$. The UVCS measurements (Kohl et al. 1998; Cranmer et al. 2000) indicate oxygen and hydrogen mean thermal speeds that are in excess of the canonical value of about $150 \mathrm{~km} / \mathrm{s}$ corresponding to $1 \mathrm{MK}$. In coronal holes strong heating of hydrogen (protons) was found with $T_{p}=2-3 \mathrm{MK}$. The oxygen ions showed even stronger (mostly perpendicular) heating, leading to $T_{o} \leq 100 \mathrm{MK}$. A large temperature anisotropy, with $T_{o \perp} / T_{o \|}>10$, was also found. As the possible origin of this heating ioncyclotron resonance is commonly assumed (for a review see Hollweg \& Isenberg 2002). There may be many other waves involved, though, which could be absorbed through Landau damping, an old idea going back to Barnes (1969).

There is a variety of kinetic plasma waves that may be relevant in the corona: Ion cyclotron waves, with $\omega \leq \Omega_{i}=e_{i} B /\left(m_{i} c\right)$, and kinetic Alfvén waves; or whistler waves, with $\omega \leq \Omega_{e}=e_{e} B /\left(m_{e} c\right)$. In addition, for oblique propagation there might be upper and lower hybrid waves. The standard dispersion relations (at a given location in the nonuniform corona) for parallel propagating plasma waves are given in Figure 3. For further details see any space-plasma text book, e.g. Treumann \& Baumjohann (1998).

The wave absorption coefficient (or growth rate in case of an instability), i.e. the wave opacity sensitively depends upon the ion and electron velocity distributions. The heating and acceleration of the ions depend further on the unknown wave spectrum in the corona. The corresponding rates based on quasilinear theory have been calculated by Marsch \& Tu (2001) and Marsch (2002), and are discussed below. What processes could generate ion-cyclotron waves? Theoreti- 


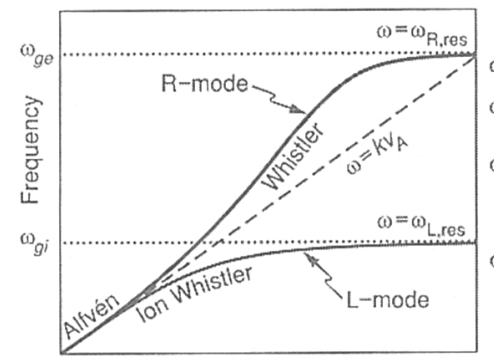

Parallel Wavenumber

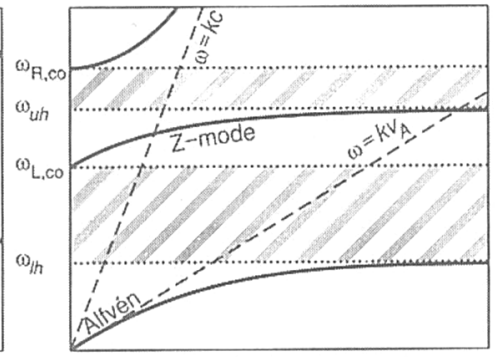

Perpendicular Wavenumber

Figure 3. Dispersion relations for parallel propagating low-frequency plasma waves (after Treumann \& Baumjohann (1998)

cally, an ion core-temperature anisotropy can drive the waves unstable, similarly hot ion beams (coronal ion jets), electric currents, e.g. due to an ion-electron drift, ion loss-cone distributions, the parametric decay of large-amplitude Alfvén waves, energy cascading from the MHD regime, or inhomogeneity leading to frequency sweeping for a spatially varying $\Omega_{i}(r)$. The proton velocity distributions observed in the fast solar wind (Helios) reveal distinct nonthermal features indicating kinetic wave activity (Marsch 1991). However, do such kinetic processes really operate in the corona? We presently do not know and therefore consider the origin and level of kinetic ion waves in the solar corona an open issue.

\section{Magnetohydrodynamic waves and oscillations of loops}

\subsection{Observations and theory}

Let us return to magnetohydrodynamic waves and discuss some observations and their consequences. Detectability of coronal MHD waves requires that spatial (pixel size) and temporal (exposure/cadence) resolution be less than the wave lengths and periods considered. The spectral resolution must be sufficient to resolve Doppler shifts and broadenings (with SUMER this can be done with subpixel accuracy of a few $\mathrm{km} / \mathrm{s}$ ).

Nakariakov (2003) has put together an instructive table of some modern imagers/spectrometers on SOHO, Yohkoh and TRACE with the relevant parameters, such as spatial resolution and minimum pixel size (ranging from $0.5^{\prime \prime}$ to $4^{\prime \prime}$ ), temporal resolution and maximal cadence (from $10 \mathrm{~s}$ to $30 \mathrm{~s}$ ), and the spectral bands. TRACE first detected longitudinal waves in intensity (density) variations which were interpreted as slow magnetoacoustic waves. TRACE loop images in Fe IX $(\lambda 17.1 \mathrm{~nm})$ were taken at a $15 \mathrm{~s}$ cadence. De Moortel et al. (2000) analyzed the properties of such longitudinal oscillations and provided a statistical overview of the physical properties of many cases of longitudinal oscillations detected at the base of large coronal loops, having lengths of a fraction of a solar radius.

Frequent Doppler oscillations were also measured by the SUMER spectrometer (see the review of Wang 2003). Some typical properties are: The oscillations are mainly seen in hot $\left(T_{e}>7 \mathrm{MK}\right)$ flare lines, sometimes correlated with cool 
emissions. They are during solar activity correlated with the flux of soft x-rays and appear to be triggered by mass ejecta and shock fronts. The periods range from 5 to 25 minutes, with typical damping times of several 10 minutes. Many events re-occur while the loops still being present.

There is general agreement that low-frequency coronal MHD waves are generated and released in the photosphere, housing the prolific energy source associated with magnetoconvection. The transport and propagation of wave energy in various forms and modes is not well understood, given the nonuniformity and variability of the coronal magnetic field. Even less understood is the final conversion of ordered mechanical into random thermal energy of the particles. Understanding this irreversible energy conversion at collisional or kinetic scales is the coronal heating problem proper.

When considering compressive MHD waves, an important dynamic time scale is the transit time, $t_{s}=L / c_{s} \approx 10 L_{10} / T_{6}^{1 / 2}$ minutes $\left(L_{10}=L / 10^{10} \mathrm{~cm}\right)$, where $c_{s}$ is the sound speed. For other relevant time scales in loops see Walsh (2002), from whom we quote typical times (wave periods) for various physical processes occurring in loops: Alfvén and fast magnetosonic waves $5 \mathrm{~s}$, slow mode $200 \mathrm{~s}$, gravity induced motions $40 \mathrm{~s}$, thermal equilibration by electron heat conduction $600 \mathrm{~s}$, radiative cooling time $3000 \mathrm{~s}$, and convection $300 \mathrm{~s}$. All these parameters of course vary with height in the loop (atmosphere).

Oscillations of idealized magnetic flux tubes have been theoretically studied for a long time (see e.g. the reviews by Roberts 1985, 1991). The main restoring forces for perturbations stem from the magnetic and thermal pressures for compressive (transverse kink or longitudinal sausage) waves or the magnetic tension for incompressive waves such as torsional Alfvén waves.

Wave equations in the solar atmosphere are strongly influenced by the gravitational stratification of the matter (Roberts 1991). This leads to a cut-off at a characteristic frequency, $\omega_{\alpha}=k_{\alpha} c_{\alpha}$ (e.g., with index $\alpha=k$ for the kink and $t$ for the transverse mode). The wave vector $k_{\alpha}$ relates to the inverse of the typical gradient scale of the restoring force involved, such as gravity or buoyancy. The wave will generally have a height-dependent phase speed, $c_{\alpha}(r)$. When deriving a wave equation for the upper chromosphere and transition region, it is found that it can often be characterized by external forcing, $F_{\alpha}$, and intrinsic dispersion, and sometimes resembles a driven Klein-Gordon equation, which may be written as:

$$
\frac{\partial^{2} Q_{\alpha}}{\partial r^{2}}-\frac{1}{c_{\alpha}^{2}} \frac{\partial^{2} Q_{\alpha}}{\partial t^{2}}-k_{\alpha}^{2} Q_{\alpha}=F_{\alpha}
$$

according to, e.g. Hasan \& Kalkofen (1999) and Nakariakov (2003). The related free-wave linear dispersion shows a minimum frequency (the cut-off) and reads:

$$
\omega(k)=\sqrt{\left(k c_{\alpha}\right)^{2}+\omega_{\alpha}^{2}} .
$$

The amplitude compensated for barometric stratification is given by $Q(r, t)=$ $\xi(r, t) \exp (-r /(4 H))$, where $\xi(r, t)$ denotes the original wave amplitude (here we just consider a single component), and $H$ is the scale height. Generally, nonuniformity of the atmosphere leads to complex nonlinear wave mode-couplings; see e.g. the reviews by Roberts (1991) and Goossens (1991). 

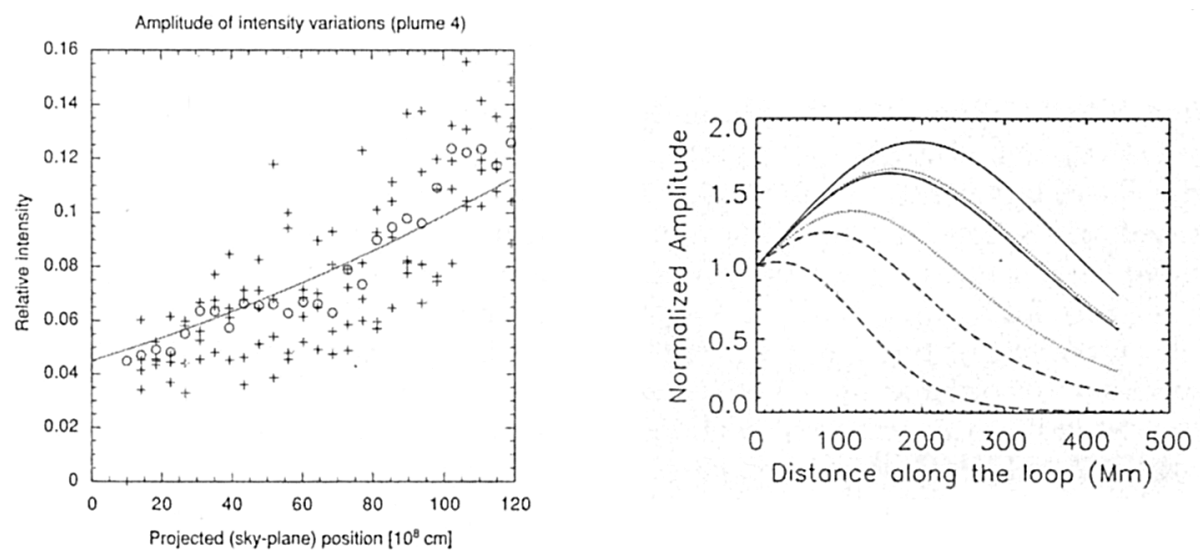

Figure 4. Left: Compressive wave amplitude relative to background in a plume (open field) versus height after Ofman et al. (1999). Right: The slow-magnetosonic wave amplitude versus height, with an initial amplitude of $\delta V_{0}=0.02 c_{s}$ in a coronal magnetic loop (closed field), after Nakariakov et al. (2000).

\subsection{Wave amplitudes versus height from numerical models}

Instead of a quasilinear analytic perturbation treatment of the MHD equations one may seek for fully nonlinear direct numerical solutions. This has in the recent past been done by various authors. Here we just give two examples for such an approach and refer to the references in the here cited literature for further work. For example, the evolution of the slow-magnetosonic wave amplitude, having an initial value of $\delta V_{0}=0.02 c_{s}$, was calculated by Nakariakov et al. (2000) as a function of height in a coronal magnetic loop. Expectedly, such models reveal strong wave steepening with height in the model atmosphere as shown in Figure 4, right frame, which indicates the steepening and subsequent limitation via disspation of the amplitude of a propagating sound wave.

Ofman et al. (1999) and coworkers have calculated numerically the turbulent outflow of the solar wind driven by MHD waves on open field lines. The nascent solar wind is strongly heated in their models by an effective viscosity and ohmic resistance, whereby the classical functional dependence of the disspation terms is retained. However, the transport coefficients are drastically enhanced by orders of magnitude over their collisional values, so as to locate the dissipation region at low heights (fraction of $R_{s}$ ). One result from their model for a polar plume in a $\mathrm{CH}$ is shown in Figure 4, left frame, showing an increasing wave amplitude versus height.

\subsection{A standing slow magnetoacoustic wave}

Many clear cases of coronal loop oscillations were associated with magnetic activity in flares. Some of the post-flare loops as seen by EIT on SOHO were studied with the help of SUMER in detail, and the results reviewed recently by Wang at al. (2003a). Figure 5, left frame, shows perhaps the clearest example 

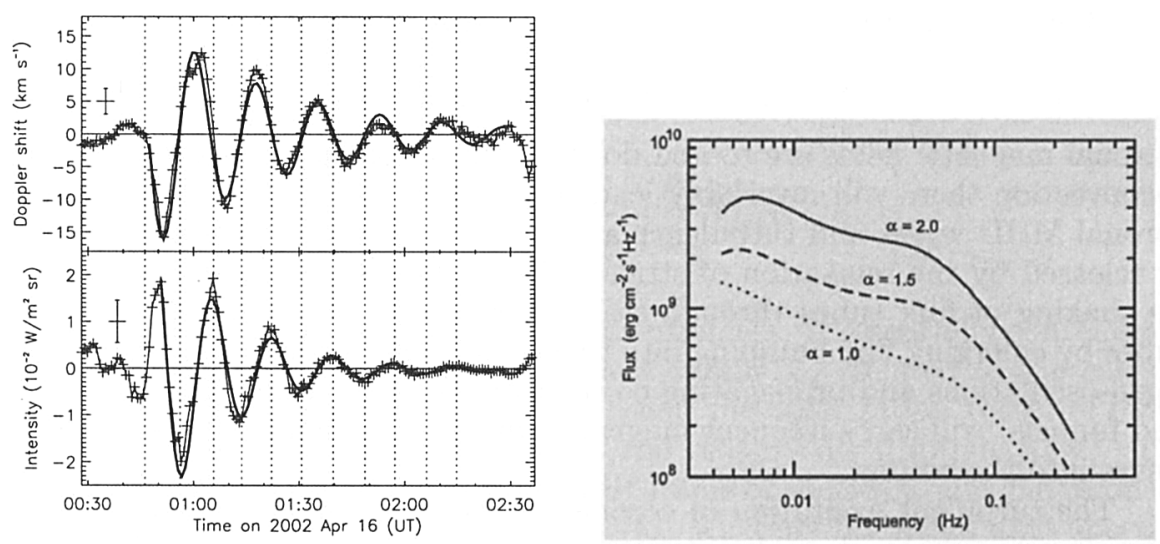

Figure 5. Left: Slow-mode standing wave detected in a post-flare loop after Wang et al. (2003b). Right: Spectral energy flux in dependence on frequency of coronal waves generated by gentle shaking of a flux tube in model calculations by Musielak \& Ulmschneider (2002).

of a standing slow-mode sound wave (Wang et al. 2003b), which was exited during a flare event. The geometrical parameters of the related loop were determined as: Azimuth angle (in East-West direction) $\alpha=19^{\circ}$, inclination angle $\theta=27^{\circ}$, angle between magnetic field and $\operatorname{LOS}$ near the slit $\gamma=15^{\circ}$, loop length $L=191 \mathrm{Mm}$, length of segment from apex (A) to foot point (F) $L_{A F}=64 \mathrm{Mm}$, with $L_{A F}=1 / 3 L$.

Doppler shift, intensity and background continuum of Fe XIX $(\lambda 111.81 \mathrm{~nm})$ were analyzed together. After removal of the background, a damped sine function was used to describe the oscillation as follows:

$$
V=V_{0}+V_{m} \sin (\omega t+\phi) \exp \left(-t / T_{d}\right)
$$

with the derived parameters: $V_{m}=181.5 \mathrm{~km} / \mathrm{s}$, period $P=17.6 \mathrm{~min}$, and damping time $T_{d}=36.8 \pm 2.6 \mathrm{~min}\left(T_{d} / P \sim 2.1\right)$. Velocity and intensity oscillations were found to have a quarter-period phase difference, pointing to slow tube-mode standing waves. Also the background continuum intensity shows quasi-periodic fluctuations, roughly in phase with the intensity oscillations in Fe XIX. With the same functional form as in equation (4), one finds for the intensity: $I_{m} / I_{0}=0.19, P=17.1 \mathrm{~min}$, and $T_{d}=21 \pm 1.6 \mathrm{~min}\left(T_{d} / P \sim 1.2\right)$.

The damping rates have been empirically determined by SUMER for some 49 cases in 27 events. The values found by Wang et al. (2003a) are in agreement with a higher dissipation rate, due to classical thermal conduction and viscosity when the temperature as seen in the post-flare loops is much higher than $10^{6} \mathrm{~K}$. The theoretical scaling relation, $T_{d} \sim P$, compares favourably with their empirical result: $T_{d} \sim 0.68 P^{1.06 \pm 0.18}$. The results obtained from TRACE for 11 cases considered by Ofman and Aschwanden (2002) are in agreement with dissipation by phase mixing for kink-mode oscillations, yielding a scaling relation $T_{d} \sim P^{4 / 3}$, which compares favourably with their empirical result: $T_{d} \sim 0.9 P^{1.30 \pm 0.21}$. 


\section{Waves and turbulence, generation and dissipation}

\subsection{Generation of magnetohydrodynamic waves}

Coronal magnetic fields are rooted down in the turbulent photosphere. Magnetoconvection there will invariably lead to waves in the corona. Most probably, coronal MHD waves and turbulence are therefore driven by magnetoconvection or released by the relaxation of strained fields through reconnection. The gentle shaking of flux tubes through steady convection or the violent pushing of tubes by emerging flux bumping into them, may lead to various kinds of coronal eigen-oscillations and propagating coronal waves. As a result, flux tubes (loops and funnels) will carry frequent magnetoacoustic waves and/or torsional Alfvén waves into the corona.

The turbulent excitation of coronal MHD waves by gentle shaking of flux tubes in the photosphere has been considered by Musielak \& Ulmschneider (2002). In a series of papers these authors provide detailed calculations of wave spectra and consider the energetics of chromospheric and coronal heating. Their model spectra are particularly relevant for a discussion of a turbulent cascade in the corona. Lack of space does not permit us to dwell on this issue. One of their model spectra is shown in Figure 5, right frame. Here $\alpha$ is a measure of the mixing length, $\ell=\alpha H$, and the barometric scale height $H$ is about $300 \mathrm{~km}$.

\subsection{Wave dissipation in the kinetic domain}

Let us now address the issue of kinetic wave dissipation. Assume that some nonlinear cascade or any instability has delivered wave energy to the kinetic scales. Any linear kinetic wave mode (index $M$ ) may be fully described by the Fourier components of its electric field vector, which is written $\tilde{\mathbf{E}}(\mathbf{k}, \omega)=$ $2 \pi \sum_{M} \delta\left(\omega-\omega_{M}(\mathbf{k})\right) E_{M}(\mathbf{k}) \mathbf{e}_{M}(\mathbf{k})$, in terms of the wave frequency, $\omega_{M}(\mathbf{k})$, unimodular polarization vector, $\mathbf{e}_{M}(\mathbf{k})$, and amplitude, $E_{M}(\mathbf{k})$. A similar expression holds for the magnetic field, which is directly given through the induction equation. The spectral energy density of the electric field of mode $M$ is given by $\mathcal{E}_{M}(\mathbf{k})=\left|E_{M}(\mathbf{k})\right|^{2} /(8 \pi)$. Marsch \& Tu (2001) have calculated the general resonant heating and acceleration rates for coronal ions and electrons by plasma waves. These volumetric rates can, for a quasi-linear superposition of waves at random phases, be cast in the compact form:

$\left(\begin{array}{c}\rho_{j} \frac{\partial}{\partial t} U_{j} \\ \rho_{j} \frac{\partial}{\partial t} V_{j \|}^{2} \\ \rho_{j} \frac{\partial}{\partial t} V_{j \perp}^{2}\end{array}\right)=\int_{-\infty}^{+\infty} \frac{d^{3} k}{(2 \pi)^{3}} \sum_{M} \frac{\mathcal{E}_{M}(\mathbf{k}) \omega_{j}^{2}}{\omega_{M}^{2}(\mathbf{k})} \sum_{s=-\infty}^{+\infty} \mathcal{R}_{j}(\mathbf{k}, s)\left(\begin{array}{c}k_{\|} \\ 2 k_{\|} w_{j}(\mathbf{k}, s) \\ s \Omega_{j}\end{array}\right)$.

Here $\omega_{j}=\left(\left(4 \pi e_{j}^{2} n_{j}\right) / m_{j}\right)^{1 / 2}$ is the plasma frequency of species $j$. The resonance function, $\mathcal{R}_{j}$, or wave opacity is a functional of the particle distribution function, $f_{j}\left(w_{\|}, w_{\perp}, t\right)$, and essentially involves its pitch-angle derivative, which is evaluated in the respective wave frame at the Landau $(s=0)$ or cyclotron (any integer Bessel function index, $s= \pm 1, \pm 2, .$.$) resonance and reads as follows:$ 


$$
\begin{array}{r}
c \mathcal{R}_{j}(\mathbf{k}, s)=-(2 \pi)^{2} \frac{k_{\|}}{\left|k_{\|}\right|} \int_{0}^{\infty} d w_{\perp} \times \\
{\left[\left|\mathbf{e}_{M}^{*}(\mathbf{k}) \cdot \mathbf{V}_{j}(\mathbf{k}, \mathbf{w}, s)\right|^{2}\left(w_{\perp} \frac{\partial}{\partial w_{\|}}+\frac{s \Omega_{j}}{k_{\|}} \frac{\partial}{\partial w_{\perp}}\right) f_{j}\left(w_{\perp}, w_{\|}\right)\right]_{w_{\|}=w_{j}(\mathbf{k}, s)}}
\end{array}
$$

This resonance function is by definition dimensionless. It essentially contains the squared scalar product between the velocity vector $\mathbf{V}_{j}$ (related to the current carried by a gyrating particle of species $j$ ), and the polarization vector of mode $M$, as well as the pitch-angle gradient of the velocity distribution $f_{j}(\mathbf{w})$. The random particle velocity $\mathbf{w}$ refers to the rest frame of species $j$, and the resonant speed is $w_{j}(\mathbf{k}, s)=\left(\omega_{M}(\mathbf{k})-k_{\|} U_{j}-s \Omega_{j}\right) / k_{\|}$, whereby the mean drift speed is denoted as $U_{j}$ and directed along the magnetic field.

To calculate the rates explicitly, one must know $f_{j}(\mathbf{w})$ and $\mathcal{E}_{M}(\mathbf{k})$ and also self-consistently solve the wave dispersion relation to obtain $\omega_{M}(\mathbf{k})$. From a theoretical point of view, assuming those quantities as being done in many models (e.g. a Maxwellian $f_{j}(\mathbf{w})$ and power-law spectrum, $\mathcal{E}_{M}(\mathbf{k}) \sim k^{-5 / 3}$ ) is not satisfying, since spectra and velocity distributions are expected to evolve substantially, quickly within a few wave damping times on small scales, and slowly owing to the inhomogeneity on large scales, as shown by Vocks and Marsch (2001). Equations (5) and (6) are rather general and apply to the corona as a dissipative linear dielectric medium permeated by weak plasma-wave turbulence.

\section{Summary and conclusion}

Recent space solar observations show that there exists a rich variety of waves on all scales, ranging from $R_{s}$ down to $\lambda_{p}$, in the magnetically structured corona. Coronal loopes were found to oscillate and flux tubes to carry kink and Alfvén waves, and slow-mode standing waves. Spectroscopic evidence was obtained for propagating waves in plumes and small-scale flows in coronal funnels. Coronal heating by cyclotron waves seems a possibility to explain the large line widths observed. Ion kinetics in the corona has in models been shown to determine the plasma-wave opacity and is expected to regulate the MHD-wave absorption. Coronal waves and turbulence are likely generated by magnetoconvection and network activity. To understand the transport properties and dissipation processes in such a multi-scale turbulent plasma is a task for future research.

\section{References}

Aschwanden, M.J., De Pontieu, B., Shrijver, C.J., \& Title, A. 2002, Solar Phys., 206, 99

Barnes, A. 1969, ApJ, 155, 311

De Moortel, I., Ireland, J., Hood, A., \& Walsh, R. 2002, Solar Phys., 209, 61

Goossens, M. 1991, in Advances in Solar System Magnetohydrodynamics, ed. E. R. Priest \& A. W. Hood, Cambridge University Press, 137

Hackenberg, A., Marsch, E., \& Mann, G. 1999, Space Sci. Rev., 87, 207 
Hansteen, V. H. 1993, ApJ, 402, 741

Hasan, S.S., \& Kalkofen, W. 1999, ApJ, 519, 899

Hassler, D.M., Dammasch, I.E., Lemaire, P., et al. 1999, Science, 283, 810

Hollweg, J.V., \& Isenberg, P.A. 2002, J. Geophys. Res., 107, SSH-12, 1

Marsch, E. 1991, in Physics of the Inner Heliosphere, Vol. II, ed. R. Schwenn \& E. Marsch, 45

Marsch, E. 2002, Nonlinear Proc. Geophys., 9, 69

Marsch, E., \& Tu, C.Y. 1997, Solar Phys., 176, 87

Marsch, E., \& Tu, C.Y. 2001, JGR, 106, 227

Marsch, E., Tu, C.Y., Wilhelm, K., Curdt, W., Schühle, U., \& Dammasch, I.E. 1997, ESA-SP-404, 555

Musielak, Z.E. \& Ulmschneider, P. 2002, A\&A, 386, 606

Ofman, L., Nakariakov, V.M., \& DeForest, C.E. 1999, ApJ, 514, 441

Ofman, L., \& Aschwanden, M.J. 2002, ApJ, 576, L153

Peter, H. 2001, A\&A, 374, 1108

Peter, H. \& Judge, P. 1999, ApJ, 522, 1148

Nakariakov, V.M., Ofman, L., \& Arber, T.D. 2000, A\&A, 353, 741

Nakariakov, V.M. 2003, in Dynamic Sun, ed. B.N. Dwivedi, Cambridge University Press, 314

Roberts, B., Edwin, P.M., \& Benz, A.O. 1984, ApJ, 279, 857

Roberts, B. 1991, in Advances in Solar System Magnetohydrodynamics, ed. E. R. Priest \& A. W. Hood, Cambridge University Press, 105

Roberts, B. 1985, in Solar System Magnetic Fields, ed. E. Priest, D. Reidel Publishing Company, 37

Treumann, R. A., \& Baumjohann, W. 1996, Basic Space Plasma Physics, Imperial College Press, London, p. 237

Seely, J.F., Feldman, U., Schühle, U., Wilhelm, K., Curdt, W., \& Lemaire, P. 1997, ApJ, 484, L87

Solanki, S.K., Lagg, A., Woch, J., Krupp, N., Collados, M. 2003, Nature, 425, 692

Tu, C. Y., Marsch, E. \& Wilhelm, K. \& Curdt, W. 1998, ApJ, 503, 475

Tu, C. Y., Marsch, E. \& Wilhelm, K. 1999, Space Sci. Rev., 87, 331

Vocks, C., \& Marsch, E. 2001, ApJ, 568, 1030

Walsh, R. 2002, ESA SP-508, 253

Wang, T.J., Solanki, S.K., Curdt, W., Innes, D.E., Dammasch, I.E., \& Kliem, B. 2003a, A\&A, 406, 1105

Wang, T.J., Solanki, S.K., Innes, D.E., Curdt. W., \& Marsch, E. 2003b, A\&A, 402, L17

Wang, T.J. 2003, ESA SP-547, 1

Wilhelm, K., Marsch, E., Dviwedi, B.N., Hassler, D.M., Lemaire, P., Gabriel, A., \& Huber, M.C.E. 1998, ApJ, 500, 1023

Wilhelm, K., Dammasch, I.E., Marsch, E., \& Hassler, D. 2000, A\&A, 353, 749

Xia, L. Marsch, E., \& Curdt, W. 2003, A\&A, 399, L5 